\title{
Kinematic Analysis and Synthesis of a 3-URU Pure Rotational Parallel Mechanism with Respect to Singularity and Workspace*
}

\author{
Syamsul HUDA $^{* *}$ and Yukio TAKEDA** \\ ** Department of Mechanical Sciences and Engineering, Tokyo Institute of Technology \\ 2-12-1 O-okayakma, Meguro-Ku, Tokyo 152-8552 Japan \\ E-mail:syamsul@stu.mech.titech.ac.jp, takeda@mech.titech.ac.jp
}

\begin{abstract}
This paper concerns kinematics and dimensional synthesis of a three universal-revolute-universal (3-URU) pure rotational parallel mechanism. The mechanism is composed of a base, a platform and three symmetric limbs consisting of U-R-U joints. This mechanism is a spatial non-overconstrained mechanism with three degrees of freedom. The joints in each limb are so arranged to perform pure rotational motion of the platform around a specific point. Equations for inverse displacement analysis and singularities were derived to investigate the relationship of the kinematic constants to the solution of the inverse kinematics and singularities. Based on the results, a dimensional synthesis procedure for the 3-URU parallel mechanism considering singularities and the workspace was proposed. A numerical example was also presented to illustrate the synthesis method.
\end{abstract}

Key words: Robotics, Lower-dof Parallel Mechanism, Kinematics, Dimensional Synthesis, Constraint Singularity, Actuation Singularity, Workspace

\section{Introduction}

A parallel mechanism features two rigid bodies: a movable body (platform) and a fixed base (base). The platform and base are connected by several kinematic chains (limbs). Recent robotic researches have shown increasing interests in spatial parallel mechanisms with fewer than six degrees of freedom (dof). Such a mechanism is called a lower-dof parallel mechanism. It is hoped that a lower-dof parallel mechanism will have a simpler structure and perform a task with fewer actuators. Besides these hoped for advantages, some lower-dof parallel mechanisms can perform pure rotational or translational motions of the platform for certain applications. A parallel mechanism performing pure rotational motion of the platform (pure rotational parallel mechanism), which is a lower-dof parallel mechanism, can be used in some applications such as orienting devices, machine tools, and medical equipment.

Many researchers have investigated pure rotational parallel mechanisms. The optimum design of a spherical parallel mechanism with three dof, which is composed of 3-3R (three limbs, each of which has three revolute joints), has been considered for output isotropy ${ }^{(1)}$, motion transmissibility ${ }^{(2)}$, and output isotropy and stiffness ${ }^{(3)}$. Attention should also be paid to error management in designing, manufacturing, and assembling this mechanism because it is an overconstrained mechanism. Static analysis has also been done taking account of elastic deformation of links ${ }^{(2)}$. Structural synthesis of three dof asymmetrical non-overconstrained parallel mechanism has been done ${ }^{(4)}$. Gregorio ${ }^{(5)}$ proposed a new 3-URC (universal, revolute, and cylindrical joints in a limb) pure rotational parallel

*Received 31 Oct., 2006 (№. 06-0175) [DOI: 10.1299/jamdsm.1.81] 
mechanism. Type synthesis of pure rotational parallel mechanisms was carried out by Kong (6).

One of the principal drawbacks of parallel mechanisms is the presence of singularities in the workspace. For lower-dof parallel mechanisms, actuation singularity ${ }^{(7)-(12)}$, constraint singularity ${ }^{(13)-(19)}$, and limb singularity (presence of local mobility in a limb) ${ }^{(20),(21)}$ should be considered in dimensional synthesis. Zlatanov ${ }^{(15)}$ proposed a 3-URU parallel mechanism that performs different platform motions, such as pure translational motion, pure rotational motion and, planar motion with three dof, without disassembly by transiting between modes of operation through a constraint singularity. The present paper focuses on dimensional synthesis of a 3-URU pure rotational parallel mechanism by taking account of singularities.

This paper is organized as follows. Section 2 describes the 3-URU pure rotational parallel mechanism's configuration, kinematic constants, joint screws, and their reciprocal screws. Section 3 derives equations for inverse displacement analysis. Section 4 derives equations for singularity analysis. The kinematic constants that affect the singularity, the position of the rotation center of the platform, and the workspace are then investigated. Based on these results, a dimensional synthesis procedure is proposed in section 5. Section 6 presents a numerical example introducing singularity maps to help in the dimensional synthesis of the mechanism.

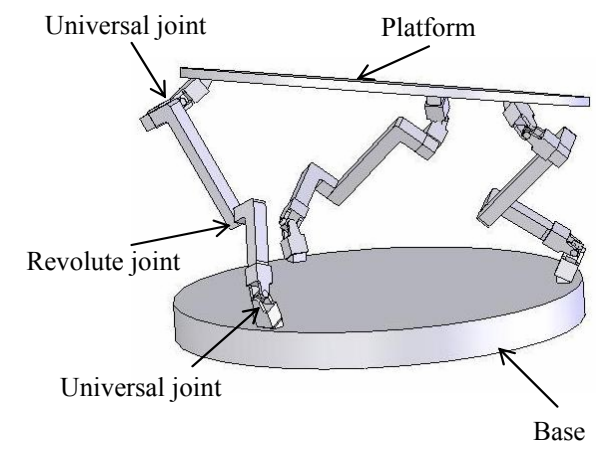

Fig. 1 CAD model of 3-URU pure rotational parallel mechanism

\section{Nomenclatures}

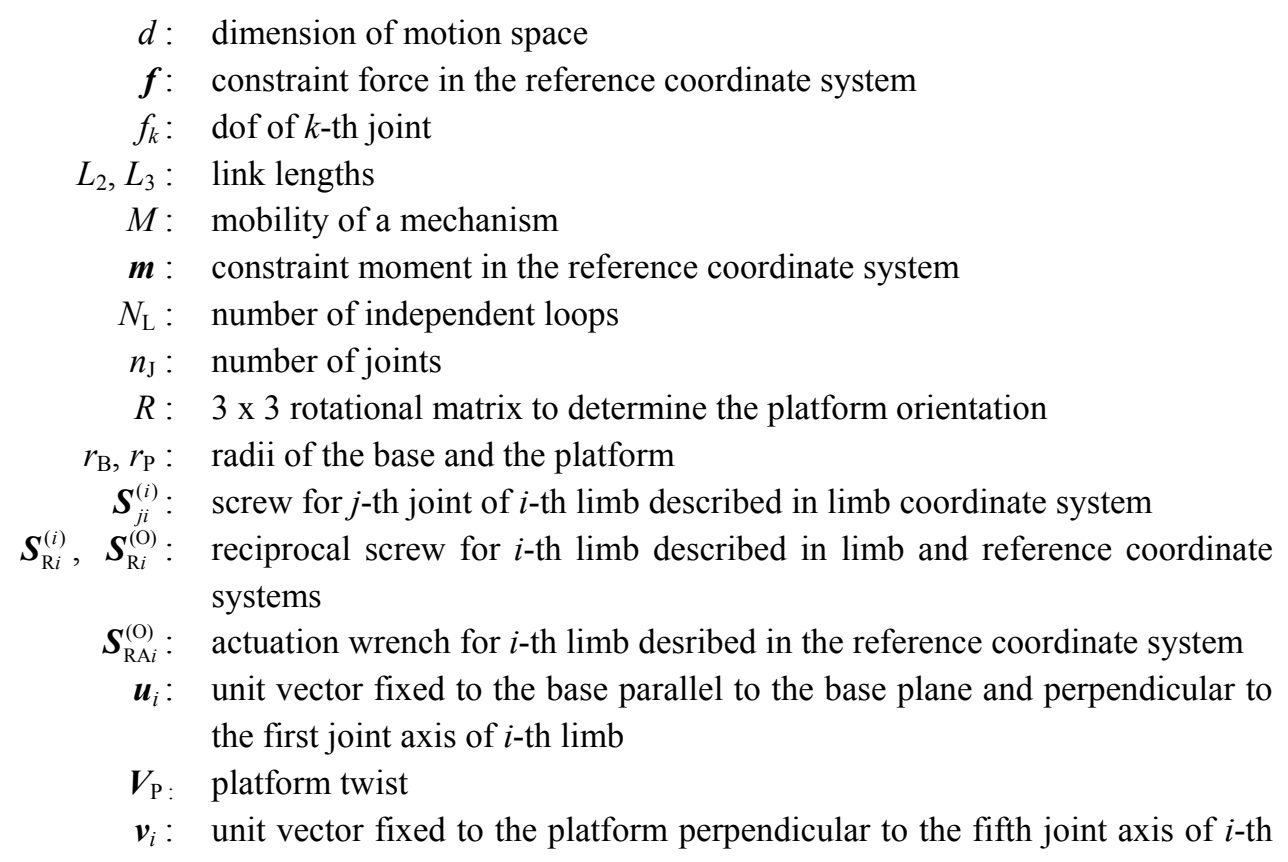




\section{$\operatorname{limb}$}

$\boldsymbol{w}_{j i}: \quad$ vector to represent the axis direction of $j$-th joint of $i$-th limb

$\lambda_{2}, \lambda_{3}$ : angle to determine the location of the second and third limbs on the base and platform

$\tau_{i}$ : magnitude of constraint wrench imposed by $i$-th limb to the platform

$\theta_{j i}: \quad$ joint displacement $j$-th joint of $i$-th limb

$\dot{\theta}_{j i}$ : angular velocity of $j$-th joint of $i$-th limb

$\psi:$ angle of the first revolute joint relative to the base plane

$\omega_{\mathrm{P}}$ : platform angular velocity

$\xi_{\mathrm{z}}, \xi_{\mathrm{x}}, \phi:$ angles to represent the platform orientation

$\zeta: \quad$ angle of the axis of the fifth revolute joint attached to the platform

\section{3-URU Pure Rotational Parallel Mechanism}

\subsection{Mechanism Configuration}

Figure 1 shows a CAD model of a 3-URU pure rotational parallel mechanism. The mechanism consists of a base, a platform, and three symmetric limbs connecting them. Each limb has two universal (U) joints and one revolute (R) joint. The kinematic diagram of the mechanism (Fig. 2) shows the arrangement of joints and links to obtain a pure rotational motion of the platform. The arrangement is based on the conditions reported by Kong and Gosselin ${ }^{(6)}$, where each limb has three parallel axes and two intersecting axes to perform such a motion. This mechanism is a special case of a non-overconstrained 3-5R pure rotational parallel mechanism, which is composed of three limbs with five revolute joints, whereby the link lengths between the first and second joints and between the fourth and fifth joints of a $5 \mathrm{R}$ limb are set to zero. In the following, we consider that each limb is composed of five revolute joints, decomposing each universal joint into two revolute joints. In Fig. 2, $\mathrm{A}_{i}$ and $\mathrm{C}_{i}(i=1,2,3)$ are the points of intersection of the first and second revolute joints and of the fourth and fifth revolute joints of the $i$-th limb. The joints of each limb are arranged so that the axis of the first revolute joint, $\boldsymbol{w}_{1 i}$, and the axis of the fifth revolute joint, $\boldsymbol{w}_{5 i}$, intersect at the center of the rotation of the platform, a point $\mathrm{O}$, and the axes of the second to fourth revolute joints, $\boldsymbol{w}_{2 i}$ to $\boldsymbol{w}_{4 i}$, are parallel to each other.

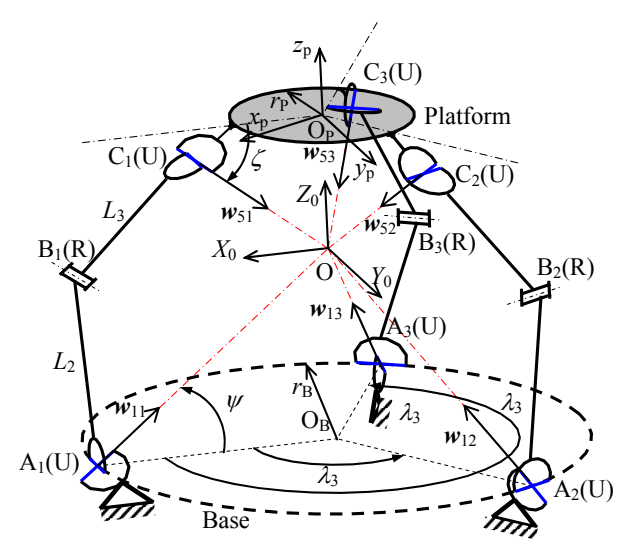

Fig. 2 Kinematic constants

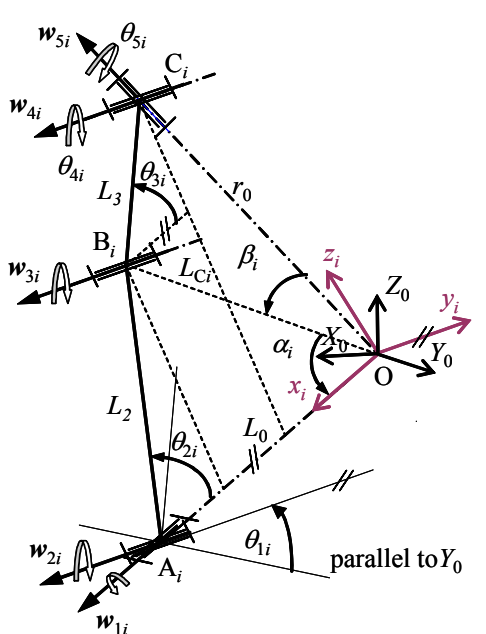

Fig. 3 Definitions of the moving coordinate system $\mathrm{O}-x_{i} y_{i} z_{i}$ and joint displacements of the $i$-th limb 
Based on the following mobility equation of a mechanism expressed as

$$
M=\sum_{k=1}^{n_{J}} f_{k}-d N_{\mathrm{L}}
$$

we can confirm that the 3-URU parallel mechanism has three dof $(M=3)$, by substituting $d$ $=6, n_{\mathrm{J}}=15, f_{k}=1(k=1,2, \ldots, 15), N_{\mathrm{L}}=2$ into Eq. (1). We consider a mechanism in which three revolute joints connected to the base (the first revolute joint of each limb) are active joints.

\subsection{Kinematic Constants}

We consider a 3-URU pure rotational parallel mechanism with a symmetric arrangement of limbs and identical limb structures. The kinematic constants of the 3-URU mechanism are classified into two groups. One group is to determine the position and orientation of universal joints on the base and the platform, that is the angles $\psi, \zeta$ and $\lambda$ and, radii $r_{\mathrm{B}}$ and $r_{\mathrm{P}}$ shown in Fig. 2. The other group contains lengths of two links of each limb, that is, $L_{2}$ and $L_{3}$. All these kinematic constants are determined in the dimensional synthesis of the mechanism.

The position of the center of platform rotation $\mathrm{O}$ is determined by $\psi, \zeta, r_{\mathrm{B}}$, and $r_{\mathrm{P}}$. As shown in Fig. 2, we define two coordinate systems. One is the reference coordinate system $\mathrm{O}-X_{0} Y_{0} Z_{0}$ fixed to the base, where the base plane is parallel to the $X_{0} Y_{0}$ plane. The other is a moving coordinate system $\mathrm{O}_{\mathrm{P}}-x_{\mathrm{P}} y_{\mathrm{P}} z_{\mathrm{P}}$ fixed to the platform, where the platform plane is parallel to the $x_{\mathrm{P}} y_{\mathrm{P}}$ plane and the $x_{\mathrm{P}}$ axis is parallel to $\overrightarrow{\mathrm{O}_{\mathrm{P}} \mathrm{C}_{1}}$.

\subsection{Joint Screws and their Reciprocal Screws}

Figure 3 shows a coordinate system $\mathrm{O}-x_{i} y_{i} z_{i}$ which moves with the motion of the first revolute joint of the $i$-th limb. The displacement of the $j$-th joint of the $i$-th limb is denoted as $\theta_{j i}$. The relationship between the moving coordinate system $\mathrm{O}-x_{i} y_{i} z_{i}$ and the reference coordinate system is expressed as follows:

$$
\left[\begin{array}{c}
X_{0} \\
Y_{0} \\
Z_{0}
\end{array}\right]=R(\psi) R\left(\theta_{1 i}\right)\left[\begin{array}{c}
x_{i} \\
y_{i} \\
z_{i}
\end{array}\right], R(\psi)=\left[\begin{array}{ccc}
\cos \psi & 0 & \sin \psi \\
0 & 1 & 0 \\
-\sin \psi & 0 & \cos \psi
\end{array}\right], R\left(\theta_{1 i}\right)=\left[\begin{array}{ccc}
1 & 0 & 0 \\
0 & \cos \theta_{1 i} & -\sin \theta_{1 i} \\
0 & \sin \theta_{1 i} & \cos \theta_{1 i}
\end{array}\right] .
$$

From Fig. 3, we obtain the screws of the five joints of each limb with respect to $\mathrm{O}-x_{i} y_{i} z_{i}$ as:

$$
\left.\begin{array}{rl}
\boldsymbol{S}_{1 i}^{(\mathrm{i})} & =\left[\begin{array}{llllll}
1 & 0 & 0 & 0 & 0 & 0
\end{array}\right]^{\mathrm{T}} \\
\boldsymbol{S}_{2 i}^{(i)} & =\left[\begin{array}{llllll}
0 & -1 & 0 & 0 & 0 & a_{26}
\end{array}\right]^{\mathrm{T}} \\
\boldsymbol{S}_{3 i}^{(i)} & =\left[\begin{array}{llllll}
0 & -1 & 0 & a_{34} & 0 & a_{36}
\end{array}\right]^{\mathrm{T}} \\
\boldsymbol{S}_{4 i}^{(i)} & =\left[\begin{array}{llllll}
0 & -1 & 0 & a_{44} & 0 & a_{46}
\end{array}\right]^{\mathrm{T}} \\
\boldsymbol{S}_{5 i}^{(i)} & =\left[\begin{array}{llllll}
a_{51} & 0 & a_{53} & 0 & 0 & 0
\end{array}\right]^{\mathrm{T}}
\end{array}\right\},
$$

where $a_{26}=-L_{0}, a_{34}=L_{2} \sin \theta_{2 i}, a_{36}=-\left\{L_{0}-L_{2} \cos \theta_{2 i}\right\}, a_{44}=L_{2} \sin \theta_{2 i}+L_{3} \sin \theta_{3 i}$, $a_{46}=-\left\{L_{0}-L_{2} \cos \theta_{2 i}-L_{3} \cos \theta_{3 i}\right\}, \quad a_{51}=\cos \left(\alpha_{i}+\beta_{i}\right), \quad a_{53}=\sin \left(\alpha_{i}+\beta_{i}\right), \quad L_{0}=r_{\mathrm{B}} / \cos \psi$, $r_{0}=r_{\mathrm{P}} / \cos \zeta \quad, \quad L_{\mathrm{C} i}=\sqrt{L_{0}^{2}+L_{2}^{2}-2 L_{0} L_{2} \cos \theta_{2 i}} \quad, \quad \alpha_{i}=\cos ^{-1}\left\{\left(L_{0}^{2}+L_{\mathrm{C} i}^{2}-L_{2}^{2}\right) /\left(2 L_{0} L_{\mathrm{C} i}\right)\right\} \quad$, $\beta_{i}=\cos ^{-1}\left\{\left(r_{0}^{2}+L_{\mathrm{C} i}^{2}-L_{3}^{2}\right) /\left(2 r_{0} L_{\mathrm{C} i}\right)\right\}$. The superscript $(i)$ of a screw indicates that it is described in $\mathrm{O}-x_{i} y_{i} z_{i}$.

For all five screws of each limb in Eq. (3), we can obtain a reciprocal screw, $\boldsymbol{S}_{\mathrm{R} i}$, that represents the constraint imposed to the platform by the limb. $\boldsymbol{S}_{\mathrm{R} i}$ is obtained by solving the following equations.

$$
\boldsymbol{S}_{\mathrm{R} i} \circ \boldsymbol{S}_{j i}=0
$$


Here, the operation $\circ$ represents the reciprocal product of screws ${ }^{(22)}$.

Using Eqs. (3) and (4), the reciprocal screw of the first limb, $\boldsymbol{S}_{\mathrm{R} 1}^{(1)}$ with respect to the $\mathrm{O}-x_{i} y_{i} z_{i}$ coordinate system, is expressed as

$$
\boldsymbol{S}_{\mathrm{R} 1}^{(1)}=\left[\begin{array}{llllll}
0 & 1 & 0 & 0 & 0 & 0
\end{array}\right]^{\mathrm{T}} .
$$

Using Eq. (2), the expression of the reciprocal screw, $\boldsymbol{S}_{\mathrm{R} 1}^{(1)}$ in Eq. (5), in terms of the reference coordinate system $\mathrm{O}-X_{0} Y_{0} Z_{0}, \quad \boldsymbol{S}_{\mathrm{R} 1}^{(\mathrm{O})}$, can be written as:

$$
\boldsymbol{S}_{\mathrm{R} 1}^{(\mathrm{O})}=\left[\begin{array}{llllll}
\sin \psi \sin \theta_{11} & \cos \theta_{11} & \cos \psi \sin \theta_{11} & 0 & 0 & 0
\end{array}\right]^{\mathrm{T}}
$$

Here, the superscript $(\mathrm{O})$ of a screw indicates that it is described in $\mathrm{O}-X_{0} Y_{0} Z_{0}$. Two other reciprocal screws of the second and third limbs, $\boldsymbol{S}_{\mathrm{R} 2}^{(\mathrm{O})}$ and $\boldsymbol{S}_{\mathrm{R} 3}^{(\mathrm{O})}$, are determined as follows:

$$
\boldsymbol{S}_{\mathrm{R} i}^{(\mathrm{O})}=\left[\begin{array}{cc}
R\left(\lambda_{i}\right) & 0_{3 \times 3} \\
0_{3 \times 3} & R\left(\lambda_{i}\right)
\end{array}\right] \boldsymbol{S}_{\mathrm{R} 1}^{(\mathrm{O})}, \quad R\left(\lambda_{i}\right)=\left[\begin{array}{ccc}
\cos \lambda_{i} & -\sin \lambda_{i} & 0 \\
\sin \lambda_{i} & \cos \lambda_{i} & 0 \\
0 & 0 & 1
\end{array}\right](i=2 \text { and } 3)
$$

Here, $0_{(3 \times 3)}$ is a $3 \times 3$ zero matrix. Substituting $\lambda_{1}=-2 \pi / 3 \mathrm{rad}$ and $\lambda_{2}=-4 \pi / 3 \mathrm{rad}$ into Eq. (7), we obtain reciprocal screws $\boldsymbol{S}_{\mathrm{R} 2}^{(\mathrm{O})}$ and $\boldsymbol{S}_{\mathrm{R} 3}^{(\mathrm{O})}$ :

$$
\left.\begin{array}{l}
\boldsymbol{S}_{\mathrm{R} 2}^{(\mathrm{O})}=\left[\begin{array}{c}
\boldsymbol{s}_{\mathrm{R} 2}^{(\mathrm{O})} \\
\mathbf{0}
\end{array}\right], \quad \boldsymbol{S}_{\mathrm{R} 3}^{(\mathrm{O})}=\left[\begin{array}{c}
\boldsymbol{s}_{\mathrm{R} 3}^{(\mathrm{O})} \\
\mathbf{0}
\end{array}\right], \\
\boldsymbol{s}_{\mathrm{R} 2}^{(\mathrm{O})}=\left[\begin{array}{lll}
-\frac{\sqrt{3}}{2} \cos \theta_{12}-\frac{1}{2} \sin \psi \sin \theta_{12} & -\frac{1}{2} \cos \theta_{12}+\frac{\sqrt{3}}{2} \sin \psi \sin \theta_{12} & \cos \psi \sin \theta_{12}
\end{array}\right]^{\mathrm{T}}, \\
\boldsymbol{s}_{\mathrm{R} 3}^{(\mathrm{O})}=\left[\begin{array}{lll}
\frac{\sqrt{3}}{2} \cos \theta_{13}-\frac{1}{2} \sin \psi \sin \theta_{13} & -\frac{1}{2} \cos \theta_{13}-\frac{\sqrt{3}}{2} \sin \psi \sin \theta_{13} & \cos \psi \sin \theta_{13}
\end{array}\right]^{\mathrm{T}},
\end{array}\right\}
$$

where $\mathbf{0}$ represents a three-dimensional zero vector.

\subsection{Platform Motion}

From Eqs. (6) and (8), the wrench $\boldsymbol{F}=\left[\begin{array}{ll}\boldsymbol{f}^{\mathrm{T}} & \boldsymbol{m}^{\mathrm{T}}\end{array}\right]^{\mathrm{T}}$ imposed to the platform by the three limbs as a constraint can be obtained as:

$$
\begin{aligned}
\boldsymbol{F} & =\left[\begin{array}{c}
Q \\
0_{(3 \times 3)}
\end{array}\right] \tau \\
Q & =\left[\begin{array}{ccc}
\sin \psi \sin \theta_{11} & -\frac{\sqrt{3}}{2} \cos \theta_{12}-\frac{1}{2} \sin \psi \sin \theta_{12} & \frac{\sqrt{3}}{2} \cos \theta_{13}-\frac{1}{2} \sin \psi \sin \theta_{13} \\
\cos \theta_{11} & -\frac{1}{2} \cos \theta_{12}+\frac{\sqrt{3}}{2} \sin \psi \sin \theta_{12} & -\frac{1}{2} \cos \theta_{13}-\frac{\sqrt{3}}{2} \sin \psi \sin \theta_{13} \\
\cos \psi \sin \theta_{11} & \cos \psi \sin \theta_{12} & \cos \psi \sin \theta_{13}
\end{array}\right]
\end{aligned}
$$

where $\boldsymbol{f}=\left[\begin{array}{lll}f_{X} & f_{Y} & f_{Z}\end{array}\right]^{\mathrm{T}}$ and $\boldsymbol{m}=\left[\begin{array}{lll}m_{X} & m_{Y} & m_{Z}\end{array}\right]^{\mathrm{T}}$ and $\boldsymbol{\tau}=\left[\begin{array}{lll}\tau_{1} & \tau_{2} & \tau_{3}\end{array}\right]^{\mathrm{T}}$. From Eq. (9), it is known that there are no moment constraints about $\mathrm{O}$ imposed by the three limbs to the platform. This means that any rotational motion of platform around $\mathrm{O}$ can be achieved. If and only if the matrix $Q$ in Eq. (10) does not vanish, the three constraints imposed by the three limbs are independent of each other, and translational motion of the platform is completely constrained. The discussion here indicates that the 3-URU parallel mechanism can perform pure rotational motion of the platform around $\mathrm{O}$ if $Q$ in Eq. (10) does not vanish.

\section{Displacement Analysis}

Equations are derived for inverse displacement calculation, for which the orientation of the platform is given and joint displacements $\theta_{1 i}$ to $\theta_{5 i}$ are obtained.

\subsection{Representation of Platform Orientation}




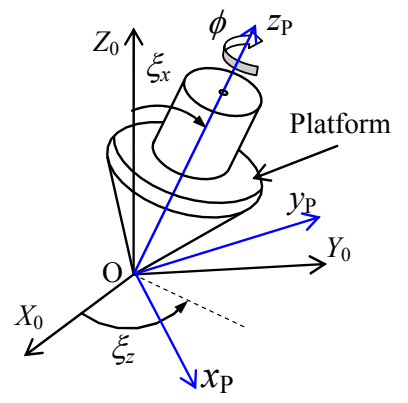

Fig. 4 Definition of platform orientation

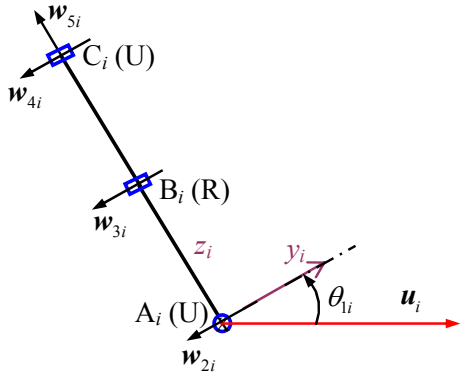

(a) in $y_{i} z_{i}$ plane

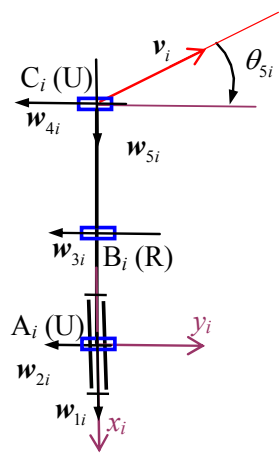

(b) in $x_{i} y_{i}$ plane

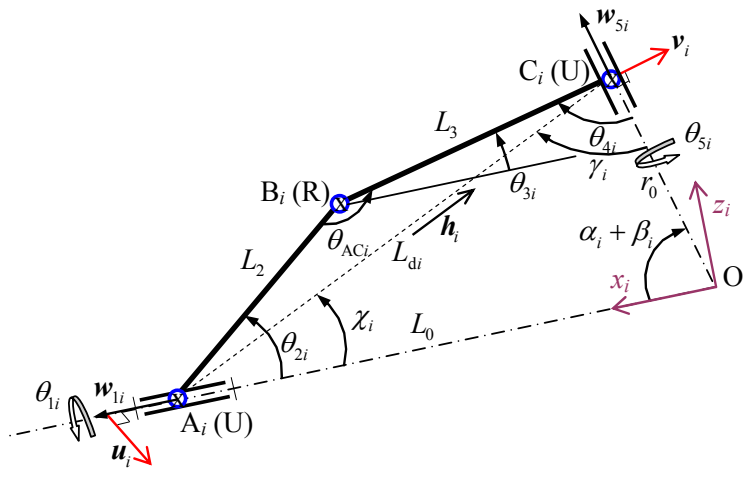

(c) in $x_{i} z_{i}$ plane

Fig. 5 Representation of a limb

The platform orientation with respect to the base is defined by three parameters shown in Fig. 4. They consist of successive rotations of $Z\left(\xi_{\mathrm{z}}\right)-X\left(\xi_{\mathrm{x}}\right)-Z(\phi)$ system. If we denote a vector in $\mathrm{O}-X_{0} Y_{0} Z_{0}$ by $\boldsymbol{p}_{\mathrm{O}}=\left[\begin{array}{lll}X & Y & Z\end{array}\right]^{\mathrm{T}}$ and a vector in $\mathrm{O}_{\mathrm{P}}-x_{\mathrm{P}} y_{\mathrm{P}} z_{\mathrm{P}}$ by $\boldsymbol{p}=\left[\begin{array}{lll}x & y & z\end{array}\right]^{\mathrm{T}}$, we can obtain the following relationship between $\boldsymbol{p}_{\mathrm{O}}$ and $\boldsymbol{p}$,

$$
\boldsymbol{p}_{\mathrm{O}}=R \boldsymbol{p}
$$

where $R$ is the rotation matrix written as

$$
R=\left[\begin{array}{ccc}
\mathrm{c} \xi_{z} \mathrm{c} \phi-\mathrm{s} \xi_{z} \mathrm{c} \xi_{x} \mathrm{~s} \phi & -\mathrm{c} \xi_{z} \mathrm{~s} \phi-\mathrm{s} \xi_{z} \mathrm{c} \xi_{x} \mathrm{c} \phi & \mathrm{s} \xi_{z} \mathrm{~s} \xi_{x} \\
\mathrm{~s} \xi_{z} \mathrm{c} \phi+\mathrm{c} \xi_{z} \mathrm{c} \xi_{x} \mathrm{~s} \phi & -\mathrm{s} \xi_{z} \mathrm{~s} \phi+\mathrm{c} \xi_{z} \mathrm{c} \xi_{x} \mathrm{c} \phi & -\mathrm{c} \xi_{z} \mathrm{~s} \xi_{\mathrm{x}} \\
\mathrm{s} \xi_{x} \mathrm{~s} \phi & \mathrm{s} \xi_{x} \mathrm{c} \phi & \mathrm{c} \xi_{x}
\end{array}\right],
$$

and $\mathrm{c} \xi_{\mathrm{z}}=\cos \xi_{\mathrm{z}}, \mathrm{c} \xi_{\mathrm{x}}=\cos \xi_{\mathrm{x}}, \mathrm{s} \xi_{\mathrm{z}}=\sin \xi_{\mathrm{z}}, \mathrm{s} \xi_{\mathrm{x}}=\sin \xi_{\mathrm{x}}, \mathrm{s} \phi=\sin \phi$, and $\mathrm{c} \phi=\cos \phi$.

\subsection{Formulation of Displacement Analysis}

Equations for inverse displacement analysis are obtained using the condition that the platform performs pure rotational motion around $\mathrm{O}$. There are five unknown parameters for each limb. They are $\theta_{i j}(i=1,2,3 ; j=1,2, \ldots, 5)$. Figure 5 shows kinematic parameters that are used to calculate the angular displacements of a limb. In the inverse kinematics, first we calculate the vectors $\boldsymbol{w}_{5 i}$ and $\boldsymbol{v}_{i}$ from the specified orientation of the platform.

Since $\boldsymbol{w}_{2 i}$ is perpendicular to both $\boldsymbol{w}_{1 i}$ and $\boldsymbol{w}_{5 i}, \boldsymbol{w}_{2 i}$ can be calculated with

$$
\boldsymbol{w}_{2 i}=\frac{\boldsymbol{w}_{1 i} \times \boldsymbol{w}_{5 i}}{\left\|\boldsymbol{w}_{1 i} \times \boldsymbol{w}_{5 i}\right\|} \text {. }
$$

From Fig. 5(a), the following equation is obtained:

$$
\boldsymbol{w}_{2 i}=-\boldsymbol{u}_{i} \cos \theta_{1 i}-\left(\boldsymbol{w}_{1 i} \times \boldsymbol{u}_{i}\right) \sin \theta_{1 i} .
$$

Using this equation, angular displacement of the input link $\theta_{1 i}$ can be obtained with 


$$
\left.\begin{array}{l}
\cos \theta_{1 i}=-\boldsymbol{w}_{2 i} \cdot \boldsymbol{u}_{i} \\
\sin \theta_{1 i}=\left(\boldsymbol{w}_{1 i} \times \boldsymbol{u}_{i}\right) \cdot\left(-\boldsymbol{w}_{2 i}\right)
\end{array}\right\} .
$$

Using Eq. (12) and $\boldsymbol{w}_{5 i}$ and $\boldsymbol{v}_{i}$ that are calculated from the specified platform orientation, the angle $\theta_{5 i}$ can be obtained as

$$
\left.\begin{array}{l}
\cos \theta_{5 i}=-\boldsymbol{w}_{2 i} \cdot \boldsymbol{v}_{i} \\
\sin \theta_{5 i}=-\left\{\boldsymbol{w}_{2 i} \times \boldsymbol{w}_{5 i}\right\} \cdot \boldsymbol{v}_{i}
\end{array}\right\} .
$$

Using these results, the angular displacements of other joints can be calculated as follows.

$$
\begin{aligned}
& \alpha_{i}+\beta_{i}=\cos ^{-1}\left(\boldsymbol{w}_{5 i} \cdot \boldsymbol{w}_{1 i}\right) \\
& L_{\mathrm{d} i}=L_{0}^{2}+r_{0}^{2}-2 L_{0} r_{0} \cos \left(\alpha_{i}+\beta_{i}\right) \\
& \theta_{\mathrm{AC} i}=\cos ^{-1}\left\{\left(L_{2}^{2}+L_{3}^{2}-L_{\mathrm{d} i}^{2}\right) /\left(2 L_{2} L_{3}\right)\right\} \\
& \gamma_{i}=\sin ^{-1}\left\{L_{0} \sin \left(\alpha_{i}+\beta_{i}\right) / L_{\mathrm{d} i}\right\} \\
& \chi_{i}=\sin ^{-1}\left\{r_{0} \sin \left(\alpha_{i}+\beta_{i}\right) / L_{\mathrm{d} i}\right\} \\
& \theta_{2 i}=\sin ^{-1}\left\{L_{3} \sin \theta_{\mathrm{AC} i} / L_{\mathrm{d} i}\right\}+\chi_{i} \\
& \theta_{3 i}=\left(\theta_{2 i}+\theta_{\mathrm{AC} i}\right)-\pi \\
& \theta_{4 i}=\sin ^{-1}\left\{L_{2} \sin \theta_{\mathrm{AC} i} / L_{\mathrm{d} i}\right\}+\gamma_{i}
\end{aligned}
$$

Based on the results mentioned above, the solutions of the inverse displacement analysis for $\theta_{1 i}, \theta_{2 i}, \theta_{3 i}, \theta_{4 i}$ and $\theta_{5 i}(i=1,2,3)$ are obtained by analytical and simple equations.

Equations (12), (14) and (16) indicate that the angular displacements $\theta_{1 i}$ and $\alpha_{i}+\beta_{i}$ can be determined from only $\boldsymbol{w}_{1 i}$ and $\boldsymbol{w}_{5 i}$. Therefore, these angular displacements can be determined from the platform orientation and kinematic constants defining the position and orientation of the first revolute joint on the base and the fifth revolute joint on the platform, that is, $\psi, \zeta, r_{\mathrm{B}}$ and $r_{\mathrm{P}}$, and they are independent of $L_{2}$ and $L_{3}$.

\subsection{Workspace}

The workspace of the mechanism is defined as a set of rotation angles represented by three parameters. For the 3-URU parallel mechanism, the boundary of the workspace can be specified by the angle $\left(\alpha_{i}+\beta_{i}\right)$ shown in Fig. 3, where the angle is limited by

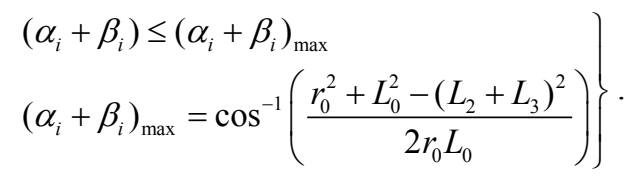

\section{Derivation of Equations for Singularities}

\subsection{Constraint Singularity}

Based on the discussion in 2.4, if the following equation is satisfied,

$$
\operatorname{det} Q=0 \text {, }
$$

the point $\mathrm{O}$ on the platform, which should be its center of rotation, can undergo translational motion because the force constraints of the three limbs become dependent. This phenomenon is called a constraint singularity. Expanding Eq. (25) using Eq. (10) yields

$$
\begin{aligned}
& \text { det } Q \\
& \begin{aligned}
=\frac{\sqrt{3}}{2} \cos \psi\left(\sin \theta_{11} \cos \theta_{12} \cos \theta_{13}+\cos \theta_{11} \sin \theta_{12} \cos \theta_{13}+\cos \theta_{11} \cos \theta_{12} \sin \theta_{13}\right. \\
\left.+3 \sin ^{2} \psi \sin \theta_{11} \sin \theta_{12} \sin \theta_{13}\right)=0 .
\end{aligned}
\end{aligned}
$$

Considering the parameters that appear in Eq. (26), we find that the condition for the 
constraint singularity is a function of $\psi$ and the input displacement, $\theta_{1 i}$. As is described in 3.2, $\theta_{1 i}$ is determined by $\boldsymbol{w}_{1 i}$ and $\boldsymbol{w}_{5 i}$, and it is independent of $L_{2}$ and $L_{3}$. Therefore, the condition of the constraint singularity is determined by only the four kinematic constants $\left(r_{\mathrm{B}}\right.$, $r_{\mathrm{P}}, \psi$ and $\zeta$ ) and the platform orientation.

\subsection{Actuation Singularity}

The instantaneous twist, $\boldsymbol{V}_{\mathrm{P}}$, of the platform can be expressed as a linear combination of instantaneous twists for five joints as

$$
\boldsymbol{V}_{\mathrm{P}}=\sum_{j=1}^{5} \boldsymbol{S}_{j i}^{(\mathrm{O})} \dot{\theta}_{j i} \quad(i=1,2,3) .
$$

For a pure rotational parallel mechanism, the platform twist, $\boldsymbol{V}_{\mathrm{P}}$, can be written as

$$
\boldsymbol{V}_{\mathrm{P}}=\left[\begin{array}{c}
\omega_{\mathrm{P}} \\
\mathbf{0}
\end{array}\right]
$$

Passive joint velocities should be eliminated from Eqs. (27) and (28) to obtain the relationship between $\dot{\theta}_{1 i}$ and $\omega_{\text {p }}$. To this end, we consider a screw, $\boldsymbol{S}_{\mathrm{RA} i}$, which is reciprocal to all passive joint screws, $\boldsymbol{S}_{2 i}$ to $\boldsymbol{S}_{5 i}$, but is not reciprocal to the joint screw of the input joint, $\boldsymbol{S}_{1 i}$. The reciprocal screw, $\boldsymbol{S}_{\mathrm{RA} i}^{(\mathrm{O})}$, expressed in the reference coordinate system $\mathrm{O}-X_{0} Y_{0} Z_{0}$ can be obtained as

$$
\boldsymbol{S}_{\mathrm{RA} i}^{(\mathrm{O})}=\left[\begin{array}{c}
\mathbf{0} \\
\boldsymbol{s}_{\mathrm{RA} i}^{(\mathrm{O})}
\end{array}\right],
$$

where

$$
\begin{gathered}
\boldsymbol{s}_{\mathrm{RA} 1}^{(\mathrm{O})}=\left[\begin{array}{c}
\cos \psi \sin \left(\alpha_{1}+\beta_{1}\right)-\sin \psi \cos \left(\alpha_{1}+\beta_{1}\right) \cos \theta_{11} \\
\cos \left(\alpha_{1}+\beta_{1}\right) \sin \theta_{11} \\
-\sin \psi \sin \left(\alpha_{1}+\beta_{1}\right)-\cos \psi \cos \left(\alpha_{1}+\beta_{1}\right) \cos \theta_{11}
\end{array}\right], \\
\boldsymbol{s}_{\mathrm{RA} 2}^{(\mathrm{O})}=\left[\begin{array}{c}
\frac{1}{2}\left(-\cos \psi \sin \left(\alpha_{2}+\beta_{2}\right)-\cos \left(\alpha_{2}+\beta_{2}\right)\left\{\sqrt{3} \sin \theta_{12}-\sin \psi \cos \theta_{12}\right\}\right) \\
\frac{1}{2}\left(\sqrt{3} \cos \psi \sin \left(\alpha_{2}+\beta_{2}\right)-\cos \left(\alpha_{2}+\beta_{2}\right)\left\{\sin \theta_{12}+\sqrt{3} \sin \psi \cos \theta_{12}\right\}\right) \\
-\sin \psi \sin \left(\alpha_{2}+\beta_{2}\right)-\cos \psi \cos \left(\alpha_{2}+\beta_{2}\right) \cos \theta_{12}
\end{array}\right], \\
\boldsymbol{s}_{\mathrm{RA} 3}^{(\mathrm{O})}=\left[\begin{array}{c}
\frac{1}{2}\left(-\cos \psi \sin \left(\alpha_{3}+\beta_{3}\right)+\cos \left(\alpha_{3}+\beta_{3}\right)\left\{\sqrt{3} \sin \theta_{13}+\sin \psi \cos \theta_{13}\right\}\right) \\
\frac{1}{2}\left(-\sqrt{3} \cos \psi \sin \left(\alpha_{3}+\beta_{3}\right)-\cos \left(\alpha_{3}+\beta_{3}\right)\left\{\sin \theta_{13}-\sqrt{3} \sin \psi \cos \theta_{13}\right\}\right) \\
-\sin \psi \sin \left(\alpha_{3}+\beta_{3}\right)-\cos \psi \cos \left(\alpha_{3}+\beta_{3}\right) \cos \theta_{13}
\end{array}\right] .
\end{gathered}
$$

Applying the reciprocal product to both sides of Eq. (27) with $S_{\mathrm{RA} i}^{(\mathrm{O})}$ yields

$$
\boldsymbol{S}_{\mathrm{RA} i}^{(\mathrm{O})} \circ\left[\begin{array}{ll}
\boldsymbol{\omega}_{\mathrm{P}}^{\mathrm{T}} & \mathbf{0}^{\mathrm{T}}
\end{array}\right]^{\mathrm{T}}=\boldsymbol{S}_{\mathrm{RA} i}^{(\mathrm{O})} \circ \boldsymbol{S}_{1 i}^{(\mathrm{O})} \dot{\theta}_{1 i} .
$$

A set of three equations of Eq. (33) can be written in a simple form as

$$
J_{\mathrm{x}} \omega_{\mathrm{P}}=J_{\mathrm{q}} \dot{\theta}
$$

where $J_{\mathrm{x}}=\left[\begin{array}{c}\boldsymbol{s}_{\mathrm{RA1}}^{(\mathrm{O}) \mathrm{T}} \\ \boldsymbol{s}_{\mathrm{RA} 2}^{(\mathrm{O}) \mathrm{T}} \\ \boldsymbol{s}_{\mathrm{RA} 3}^{(\mathrm{O}) \mathrm{T}}\end{array}\right], \quad J_{\mathrm{q}}=\left[\begin{array}{ccc}\boldsymbol{s}_{\mathrm{RA} 1}^{(\mathrm{O}) \mathrm{T}} \cdot \boldsymbol{s}_{11} & 0 & 0 \\ 0 & \boldsymbol{s}_{\mathrm{RA} 2}^{(\mathrm{O}) \mathrm{T}} \cdot \boldsymbol{s}_{12} & 0 \\ 0 & 0 & \boldsymbol{s}_{\mathrm{RA} 3}^{(\mathrm{O}) \mathrm{T}} \cdot \boldsymbol{s}_{13}\end{array}\right], \quad \dot{\boldsymbol{\theta}}=\left[\begin{array}{lll}\dot{\theta}_{11} & \dot{\theta}_{12} & \dot{\theta}_{13}\end{array}\right]^{\mathrm{T}}$, $\boldsymbol{\omega}_{\mathrm{P}}=\left[\begin{array}{lll}\omega_{X} & \omega_{Y} & \omega_{Z}\end{array}\right]^{\mathrm{T}}, \quad \boldsymbol{s}_{11}=\left[\begin{array}{lll}\cos \psi & 0 & -\sin \psi\end{array}\right]^{\mathrm{T}}, \quad \boldsymbol{s}_{12}=\left[\begin{array}{lll}-\frac{1}{2} \cos \psi & \frac{1}{2} \sqrt{3} \cos \psi & -\sin \psi\end{array}\right]^{\mathrm{T}}$, and $\boldsymbol{s}_{13}=\left[\begin{array}{lll}-\frac{1}{2} \cos \psi & -\frac{1}{2} \sqrt{3} \cos \psi & -\sin \psi\end{array}\right]^{\mathrm{T}}$.

In general, there are two types of actuation singularity: direct kinematic and inverse kinematic singularities. The inverse kinematic singularity is at the boundary of the 
workspace, and is not troublesome from the view point of design and control of the mechanism. Hence, we consider only the direct kinematic singularity, where $\operatorname{det} J_{x}=0$. At this type of singularity, the platform gains at least one rotational dof even if all input velocities are zero. To numerically identify this type of singularity in the workspace, we use the following equation, which is derived from Eq. (34),

$$
\dot{\boldsymbol{\theta}}=J_{\mathrm{q}}^{-1} J_{\mathrm{x}} \omega_{\mathrm{P}} .
$$

According to Eq. (35), the singularity can be investigated by checking the sign of the determinant $\left(J_{\mathrm{q}}^{-1} J_{\mathrm{x}}\right)^{(12)}$. If the magnitude of the determinant is close to zero and its sign changes for small change of platform orientation, there will be expected a singular point close to the point. From Eqs. (29) to (35), we find that the condition of the actuation singularity is defined by the platform orientation and certain kinematic constants, i.e., the angles $\psi$ and $\zeta$ and the radii of the base and platform $\left(r_{\mathrm{B}}\right.$ and $\left.r_{\mathrm{P}}\right)$, the same as in the case of the constraint singularity.

\subsection{Limb Singularity}

A mechanism is located at a limb singularity if the five joint screws, $\boldsymbol{S}_{1 i}^{(i)}$ to $\boldsymbol{S}_{5 i}^{(i)}$, of each limb become linearly dependent. At a limb singularity, although the joints can move freely due to the local mobility of the limb, this motion produces no platform motion. This singularity is a point where the following equation has a solution other than $\boldsymbol{x}=\mathbf{0}$.

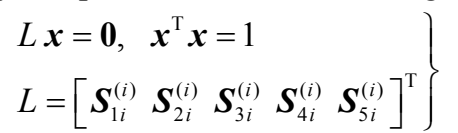

We tried to find a limb singularity of the 3-URU parallel mechanism through simulations. However, we could not find any limb singularity other than those on the boundary of the workspace, such as $\alpha_{i}+\beta_{i}=0, \pi$. Since such singular points are not troublesome in the dimensional synthesis, we do not consider the limb singularity in the following section.

\section{Dimensional Synthesis Procedure}

In the dimensional synthesis of a parallel mechanism, the values of all kinematic constants should be determined so that the mechanism properly operates in the prescribed workspace given in the design specifications. This is the most essential problem in the kinematic synthesis. For a pure rotational parallel mechanism, the prescribed workspace is specified by three parameters to represent the platform orientation, such as $\left(\xi_{\mathrm{z}}, \xi_{\mathrm{x}}, \phi\right)$ in Fig. 4. In order that the mechanism properly operates in the prescribed workspace, the following conditions should be satisfied.

(1) The reachable workspace by the mechanism should include the prescribed workspace.

(2) There are no singular points in the prescribed workspace.

The reachable workspace of the mechanism can be determined if the values of all kinematic constants are given. On the other hand, the discussion in section 4 indicates that the singular points of the 3-URU mechanism can be obtained in three-dimensional space for representing the platform orientation once the values of the kinematic constants $\psi, \zeta, r_{\mathrm{B}}$ and $r_{\mathrm{P}}$ are given. The position of the rotation center of the platform is also determined by $\psi, \zeta$, $r_{\mathrm{B}}$ and $r_{\mathrm{P}}$.

Based on the above discussion, we propose the following dimensional synthesis procedure for the 3-URU pure rotational parallel mechanism.

[Step 1] Determination of kinematic constants $\psi, \zeta, r_{\mathrm{B}}$ and $r_{\mathrm{P}}$ based on singularity conditions

Candidate sets of these kinematic constants are obtained so that singular points (constraint and actuation singularities) do not exist in the prescribed workspace. The position of the rotation center of the platform is also considered when determining these constants. 
Table 1Two candidate sets of kinematic constants of the 3-URU mechanism

\begin{tabular}{|c|c|c|}
\hline Kinematic constant & Candidate-1 & Candidate-2 \\
\hline \hline$r_{\mathrm{B}}$ & $114 \mathrm{~mm}$ & $244.16 \mathrm{~mm}$ \\
\hline$\psi$ & $0.4565 \mathrm{rad}$ & $0.2254 \mathrm{rad}$ \\
\hline$r_{\mathrm{P}}$ & $80.57 \mathrm{~mm}$ & $20.13 \mathrm{~mm}$ \\
\hline$\zeta$ & $-0.6073 \mathrm{rad}$ & $-1.2257 \mathrm{rad}$ \\
\hline
\end{tabular}

[Step 2] Determination of kinematic constants other than $\psi, \zeta, r_{\mathrm{B}}$ and $r_{\mathrm{P}}$ taking account of the reachable workspace

Sets of other kinematic constants $\left(L_{2}\right.$ and $L_{3}$ ) are obtained for each set of kinematic constants obtained in step 1 so that the reachable workspace includes the prescribed workspace.

[Step 3] Optimization of kinematic constants

Using the performance indices of the mechanism such as the transmission index ${ }^{(23)}$ or isotropy index ${ }^{(24)}$, the optimal set of kinematic constants is determined from the sets obtained in step 2.

The following section gives an example that concretely demonstrates steps 1 and 2 .

\section{Numerical Example}

We give an example of dimensional synthesis of a 3-URU mechanism. Based on the synthesis procedure described in section 5 , we define the prescribed workspace by using the platform orientation $\left(\xi_{\mathrm{z}}, \xi_{\mathrm{x}}, \phi\right)$ and the position of the rotation center of the platform. Example specifications are given below.

(1) center of the base: $\mathrm{O}_{\mathrm{B}}(0,0,-56) \mathrm{mm}$ in $\mathrm{O}-X_{0} Y_{0} Z_{0}$

(2) prescribed workspace: $0 \leq \xi_{\mathrm{z}} \leq 2 \pi, \phi=0$

To specify the center of rotation of the platform $\mathrm{O}$, we specify the center of the base $\mathrm{O}_{\mathrm{B}}$ with respect to the reference coordinate system $\mathrm{O}-X_{0} Y_{0} Z_{0}$. As for the orientation angle $\xi_{x}$, we maximize its reachable range.

[Step 1]

The example involves two candidate sets of kinematic constants shown in Table 1 to determine the singularity of the mechanism and the rotation center of the platform. Figures 6 and 7 show singularity maps for these candidates. Black and red curves represent constraint and actuation singularity curves, respectively. The non-singular area of the two candidates is shown in Table 2. From the table, we know that candidate-2 gives larger non-singular area. We thus choose candidate-2 for step 2, in which links long enough to achieve prescribed workspace are determined. If we had more sets of kinematics constants, we would have been able to obtain better candidate sets for step 2 .

[Step 2]

Using the kinematic constants $\psi, \zeta, r_{\mathrm{B}}$ and $r_{\mathrm{P}}$ chosen in step 1, we determine $L_{2}$ and $L_{3}$ for the reachable workspace to include the prescribed workspace. Here, the prescribed workspace is the same as the non-singular area in Table 2. Numerical calculations to minimize the sum of $L_{2}$ and $L_{3}$ give $L_{2}=150 \mathrm{~mm}$ and $L_{3}=160 \mathrm{~mm}$ as a solution.

\section{Conclusions}

We carried out a kinematic study to enable a dimensional synthesis of a 3-URU parallel mechanism that performs a pure rotational motion of the platform while taking account of singularities and the workspace. Our conclusions are summarized as follows.

(1) The input displacements for inverse displacement analysis of the 3-URU parallel mechanism are determined from the platform orientation and kinematic constants defining the position and orientation of the first revolute joint on the base and the fifth revolute joint on the platform, and are independent of the link lengths of the limb. 


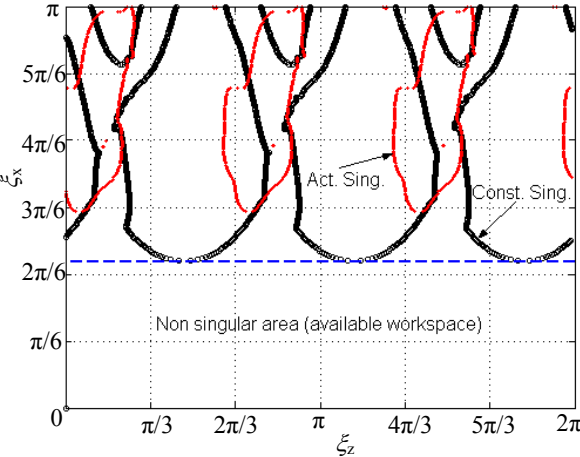

Fig. 6 Singularity map for candidate-1 $(\phi=0)$

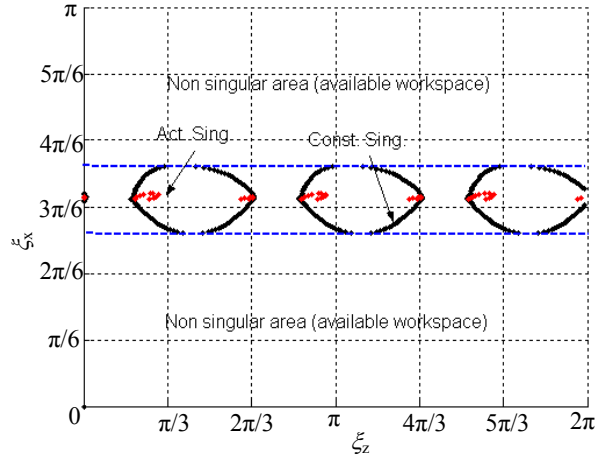

Fig. 7 Singularity map for candidate-2 $(\phi=0)$

Table 2 Non-singular area obtained in step 1 for the two candidates $(\phi=0)$

\begin{tabular}{|c|c|c|}
\hline & $\xi_{\mathrm{x}}[\mathrm{rad}]$ & $\xi_{\mathrm{z}}[\mathrm{rad}]$ \\
\hline \hline Candidate-1 & {$[0,1.15]$} & {$[0,2 \pi]$} \\
\hline Candidate-2 & {$[0,1.36],[1.89,3.14]$} & {$[0,2 \pi]$} \\
\hline
\end{tabular}

(2) The conditions of the constraint and actuation singularities of the 3-URU mechanism are determined by the kinematic constants that determine the input displacement for the inverse displacement analysis.

(3) Based on the results of (1) and (2), we proposed a dimensional synthesis method for a 3-URU mechanism that has three steps. The synthesis method divides the kinematic constants of the mechanism into two sets, one to determine the center of rotation and the singularity, and the other to determine the reachable workspace.

(4) Our proposed synthesis method simplifies the dimensional synthesis procedure of the 3-URU mechanism.

(5) A numerical example confirmed the effectiveness of our synthesis method.

\section{Acknowledgement}

This work was supported in part by a Grant-in-Aid for Scientific Research from the Ministry of Education, Culture, Sports, Science and Technology (17560116).

\section{References}

(1) Gosselin, C. and Angeles J., "The optimum design of a spherical three - degree of freedom parallel manipulators", Transactions of the ASME, Journal of mechanical Design, Vol. 111, 1989, pp. 202-207.

(2) Takeda, Y., Funabashi, H. and Sasaki, Y., "Development of a spherical in-parallel actuated mechanism with three degrees of freedom with large working space and high motion transmissibility (evaluation of motion transmissibility and analysis of working space)", JSME International Journal, Series C, Vol. 39, No.3, 1996, pp. 541-548.

(3) Liu, X., J., Jin Z., L. and Gao, F., "Optimum design of 3-dof spherical parallel manipulators with respect to the conditioning and stiffness indices", Mechanism and Machine Theory, Vol. 35, No. 9, 2000, pp. 1257-1267.

(4) Karouia, M., Herve, J. M., “Asymmetrical 3-dof spherical parallel mechanism”, European Journal of Mechanic A/solid, Vol. 24, 2005, pp. 57-67.

(5) Gregorio, R., D., "Kinematic of a new spherical parallel manipulator with three equal legs : The 3-URC wrist”, Journal of Robotic Systems, Vol.18, No. 5, 2001, pp. 213-219.

(6) Kong, X. and Gosselin C., M.,"Type synthesis of 3-dof spherical parallel manipulators based on screw theory”, Transactions of the ASME, Journal of Mechanical Design, Vol. 126, No.1, 2004, pp. 101-108. 
(7) Fichter, E., F., "A Stewart platform-based manipulator: General theory and practical construction", The International Journal of Robotics Research, Vol.5, No.2, 1986, pp.157-182.

(8) Behi, F., "Kinematic analysis for a six-degree-of-freedom 3-RPRS parallel mechanism", IEEE Journal of Robotics and Automation, Vol.4, No.5, 1988, pp.561-565.

(9) Merlet, J., P., "Singular Configurations of parallel manipulators and grassmann geometry", The International Journal of Robotics Research, Vol.8, No.5, 1989, pp.45-56.

(10) Funabashi, H., Horie, M., Kubota, T. and Takeda, Y., "Development of spatial parallel manipulators with six degrees of freedom, JSME International Journal, Series III, Vol.34, No.3, 1990, pp.382-387.

(11) Gosselin, C. and Angeles, J., "Singularity analysis of closed-loop kinematic chains", IEEE Transactions on Robotics and Automation, Vol. 6, No.3, 1990, pp.281-290.

(12) Takeda, Y. and Funabashi, H., "Kinematic and static characteristics of in-parallel actuated manipulators at singular points and in their neighbourhood", JSME International Journal, Series C, Vol.39, No.1, 1996, pp.85-93.

(13) Zlatanov, D., Bonev, I., A., Gosselin, C., M., "Constraint singularities as C-space singularities", Advances in Robot Kinematics, Kluwer Academic Publishers, 2002, pp.183-192.

(14) Zoppi, M., Bruzzone, L., E., Molfino, R., M. and Michelini, R., C., "Constraint singularities of force transmission in nonredundant parallel robots with less than six degrees of freedom", Transactions of the ASME, Journal of Mechanical Design, Vol.125, 2003, pp.557-563.

(15) Zhao, J., S., Feng, Z., J., Zhou, K. and Dong J., X., “Analysis of the singularity of spatial parallel manipulator with terminal constraints", Mechanism and Machine Theory, Vol. 40, No.3, 2005, pp.275-284.

(16) Choudhury, P. and Ghosal, A., "Singularity and controllability analysis of parallel manipulators and closed-loop mechanisms", Mechanism and Machine Theory, Vol. 35, No. 10, 2000, pp.1455-1479.

(17) Gregorio, R., D., "Kinematics of the 3-UPU wrist", Mechanism and Machine Theory, Vol. 38, No.3, 2003, pp.253-263.

(18) Bandyopahhyay, S. and Ghosal, A., "Analysis of configuration space singularities of closed-loop mechanisms and parallel manipulators", Mechanism and Machine Theory, Vol. 39, No.5, 2005, pp.519-544.

(19) Zlatanov, D., Bonev, I., A. and Gosselin, C., M., "Constraint singularities of parallel mechanisms", Proceedings of the IEEE International Conference on Robotics and Automation, 2002, pp. 496-502.

(20) Zlatanov, D., Fenton, R., G., and Benhabib, B., "Identification and classification of the singular configurations of mechanisms", Mechanisms and Machine Theory, Vol.33, No.6, 1998, pp.743-760.

(21) Takeda, Y., "Kinematic analysis of parallel mechanisms at singular points where a connecting chain has a local mobility", Mechanism and Machine Theory, Vol. 41, No.8, 2006, pp. 945-957.

(22) Sugimoto, K., "On the basis of wrench spaces for the kinematic and dynamic analysis of mechanisms", Transactions of the ASME, Journal of Mechanical Design, Vol. 125, 2003, pp. 552-556.

(23) Takeda, Y. and Funabashi, H., "Motion transmissibility of in-parallel actuated manipulators", JSME International Journal, Series C, Vol. 38, No. 4, 1995, pp. 749-755.

(24) Takeda, Y. and Funabashi, H., "Kinematic synthesis of in-parallel actuated mechanisms based on the global isotropy index", Journal of Robotics and Mechatronics, Vol. 11, No. 5, 1999, pp. 404-410. 